\title{
The effects of contextual scenes on the identification of objects
}

\author{
STEPHEN E. PALMER \\ University of California, San Diego, La Jolla, California 92037
}

\begin{abstract}
This experiment demonstrates the influence of the prior presentation of visual scenes on the identification of briefly presented drawings of real-world objects. Different pairings of objects and scenes were used to produce three main contextual conditions: appropriate, inappropriate, and no context. Correct responses and confusions with visually similar objects depended strongly on both the contextual condition and the particular target object presented. The probability of being correct was highest in the appropriate context condition and lowest in the inappropriate context condition. Confidence ratings of responses were a function of the perceptual similarity between the stimulus object and the named object; they were not strongly affected by contextual conditions. Morton's (1970 4 " $\operatorname{logogen}$ " model provided a good quantitative fit to the response probability data.
\end{abstract}

We find bread in kitchens, watches on wrists, and television sets in living rooms. People know a good deal about where objects are likely to be found in the world, and it is plausible that they use this knowledge in identifying objects they see. If so, a loaf of bread should be more easily identified when it is on a counter in the kitchen than when it is on a post in the front yard. These are context effects: effects of the environment of an object on the perception of that object, independent of the intrinsic properties of the object itself.

Many theories of perception stress this interaction of sensory and contextual information in identifying sensory input (e.g., Bruner, 1957; Morton, 1969, 1970; Neisser, 1967; Norman, 1968). While theorists differ substantially in their formulations of how context affects perception, they agree that appropriate context should aid identification and that inappropriate context should hinder it. This general result has been established in the recognition of words using sentences as contexts (e.g., Tulving \& Gold, 1963; Tulving, Mandler, \& Baumel, 1964), and in the recognition of letters using words as contexts (e.g., Reicher, 1969; Wheeler, 1970). Related effects are present in visual search phenomena. For example, less time is required to find a target object within an appropriate scene than within an inappropriate scene (Biederman, 1972; Biederman, Glass, \& Stacey, 1973). The present experiment demonstrates the importance of contextual scenes for object identification in terms of correct responses and confusions with visually

This research was supported by a Graduate Fellowship from the National Science Foundation to the author and by Research Grants NS $-07454-07$ from the National Institute of Health and GB-32235 from the National Science Foundation to Donald A Norman. The author gratefully acknowledges Kathleen $O$ 'Connor's assistance in performing the experiment and Donald A. Norman's guidance and support in all phases of this work. Additional thanks are due Norman $H$. Anderson, Lynn $A$. Cooper, and David E. Rumelhart for their helpful comments on this research and earlier drafts of this paper.

Dr. Palmer is now at the Department of Psychology, University of California, Berkeley, California 94720. similar stimuli. The paradigm also allows a quantitative test of Morton's (1969, 1970) "logogen" model for sensory-contextual interaction.

In this experiment, the context for an object is established by showing a visual scene that depicts a location where one would expect to find certain kinds of objects. The basic experimental task is illustrated in Figure 1. The subject is shown two slides: the first (left-hand drawing in Figure 1) represents a contextual scene, and the second (Drawing A, B, or C in Figure 1) depicts the target object. After a brief presentation of the target item, the subject's task is to name the object and rate his confidence in his response.

Contextual conditions are varied by different pairings of scenes with target objects. Consider, for example, the kitchen counter scene in Figure 1. When this scene is paired with the loaf of bread (Drawing A), the scene is an appropriate context for the target object. When the same scene is paired with the mailbox or drum
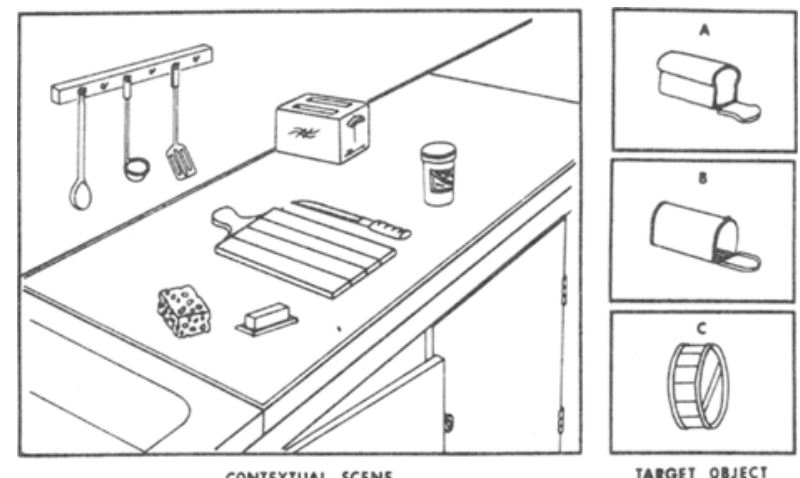

Figure 1. Examples of drawings used in the experiment. The presentation of the scene establiches the context for one of the three drawings used as target objects for this scene: a baf of bread (A), a mailbox (B), or a drum (C). See text for an explanation of contextual conditions formed by pairing scenes and objects. 
(Drawings $\mathrm{B}$ and $\mathrm{C}$ ), the scene is an inappropriate context for the target object. Additionally, when a blank scene is paired with any object, there is no context for the target object. If the contextual scenes influence the identification of objects as sentences influence the identification of words, then performance should be best where there is an appropriate context biasing responses toward the presented object, intermediate when there is no context at all, and worst when there is an inappropriate context biasing responses toward contextually appropriate objects which were not presented.

The inappropriate context condition is divided into two subconditions in order to study the interaction of sensory and contextual factors in greater detail. In one subcondition, the target object is shown after an inappropriate context, but is visually similar to an object which is appropriate to the scene. In Figure 1, for example, the mailbox (Drawing B) is inappropriate to the kitchen counter scene, but is similar in shape to the appropriate object, the loaf of bread (Drawing A). In the other subcondition, the target object is not similar to the appropriate object, as illustrated by the drum (Drawing C) when paired with the kitchen counter scene. If the target presentation is brief enough that only partial information is extracted about the target object, and if that information is consistent with the sensory properties of an appropriate object, then responses are biased toward the object which is consistent with both the sensory and contextual information available, even though a different object was actually presented. Thus, there should be fewer correct responses in the subcondition in which the target is visually similar to the contextually appropriate object than in the subcondition in which the target is not similar to the contextually appropriate object. Furthermore, more errors of naming the contextually appropriate, visually similar object are expected in the former subcondition than in the latter.

\section{Stimuli and Apparatus}

\section{METHOD}

Twelve pairs of objects were chosen so that the two items of each pair were similar in shape; one such pair is the loaf of bread and the mailbox shown as $A$ and $B$ in Figure 1. Some of the pairs of objects were approximately the same size in the real world; others were quite different in size. All objects were represented as line drawings of about the same size. For each object, a scene was drawn to provide an appropriate context. The scene did not contain the target object, but if it had, the appropriate position for the object would have been in the center of the scene.

The drawings of both scenes and target objects were projected from $35-\mathrm{mm}$ black and white negatives onto a light beige wall by a random-access projector. Thus, the pictures appeared as white line drawings on a dark gray background. The projected image of the contextual scenes subtended approximately $12 \mathrm{deg}$ of visual angle ( $2 \times 3 \mathrm{ft}$ at a distance of $10 \mathrm{ft}$ from the subjects), and the image of the target objects subtended approximately 2 deg of visual angle (averaging $3 \times 5$ in. at $10 \mathrm{ft}$ ). The exposure duration was controlled by a mechanical shutter. Dim room illumination was provided by four red lights so that subjects could see their answer sheets.

\section{Design}

Four contextual conditions were constructed by pairing objects with scenes: Appropriate context (A) in which the objects were paired with their own contextual scenes, No context $(\mathrm{N})$ in which the objects were paired with blank scenes, Inappropriate context, similar object $\left(\mathrm{I}_{\mathrm{S}}\right)$ in which the objects were paired with the scenes appropriate to the other object of the visually similar pairs, and Inappropriate context, different object $\left(I_{d}\right)$ in which the objects were paired with scenes appropriate to other objects in the experiment, but outside the visually similar pairs.

All of the 24 objects and 18 of the 24 scenes (plus six blank scenes for the no context condition) were shown to each subject. Thus, for any given subject, six objects appeared in each of the four contextual conditions. The 24 objects were divided into four "object groups" which always appeared together in the same condition. Different groups of subjects saw the object groups in different conditions, so that across all subjects, each object group (and, therefore, each object) appeared in each condition an equal number of times. This is the pairings factor: each of the four object groups was paired with one of the four contextual conditions to form a particular pairing for a group of subjects. A Latin square design was used to assign the object groups to the four contextual conditions for the four pairings required. This entire design was repeated with different groups of subjects at four exposure durations: $20,40,60$, and 120 msec.

\section{Procedure}

Written instructions were given to the subjects, informing them of their task and explaining the appropriate, inappropriate, and no context conditions. Both the written instructions and a later verbal reminder strongly encouraged the subjects to guess. Each subject was given 24 trials composed of the following sequence: (a) the presentation of a contextual scene or blank slide for $2 \mathrm{sec}$; (b) a delay of $1300 \mathrm{msec}$ while the slide changed; (c) the presentation of the target object for either $20,40,60$, or $120 \mathrm{msec}$ (always the same for any given subject); and (d) a 20 -sec interval during which each subject wrote the name of the object he or she perceived and gave a confidence rating on a five-point scale. The order of presentation for each group of subjects was randomly generated by a PDP-9 digital computer with the constraint that objects within the visually similar pairs not appear with less than six trials between them. The computer also controlled the random-access projector and the tachistoscopic shutter.

\section{Subjects}

Seventy-one students at the University of California, San Diego, participated in the experiment as part of their introductory course requirements. The data from seven subjects were eliminated: three due to equipment malfunctions, two for failing to follow instructions, and two because they forgot their glasses. All subjects were tested in groups of four or less.

\section{RESULTS AND DISCUSSION}

Each response was classified as being one of three types: the name of the presented object, the name of the other object of the visually similar pair (i.e., within-pair errors), or the name of any other object. Nonresponses were placed in the last category, but accounted for less than $0.1 \%$ of the data. All responses that were synonyms (e.g., "garbage can" and "trash can") or the names of very closely related objects (e.g., "pen" and "pencil") were classified together. From these response categories, 


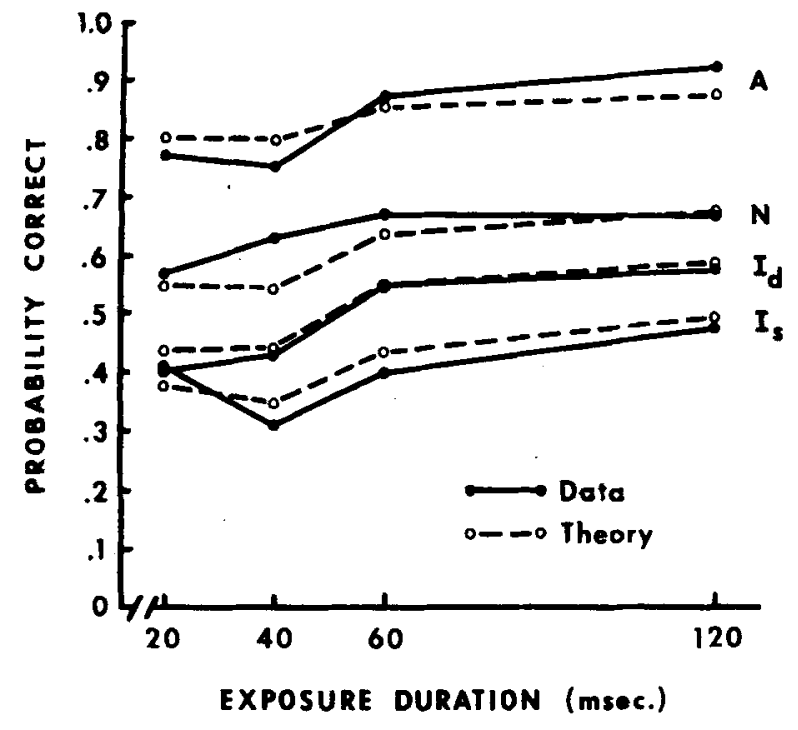

Figure 2. Probability of naming the presented object as a function of exposure duration for each contextual condition: appropriate context (A), no context $(\mathrm{N})$, inappropriate context, different object $\left(I_{d}\right)$, and inappropriate context, similar object (1). Theoretical curves represent the best fit solution for a model of contextual effects.

the probabilities of correct identifications and within-pair errors were computed.

\section{Correct Responses}

The data on correct identifications are shown in Figure 2 for all contextual conditions and exposure durations. It is evident that the probability of correctly identifying an object is highest following an appropriate context (A), lower following a blank slide $(\mathrm{N})$, and lower still following an inappropriate context $\left(\mathrm{I}_{d}\right)$ and $\left(\mathrm{I}_{\mathrm{s}}\right)$. In addition, performance in the $I_{s}$ condition (where the target is visually similar to the contextually appropriate object) is inferior to performance in the $I_{d}$ condition (where the target is visually different from the appropriate object). This follows the pattern of results obtained in similar experiments using linguistic materials (e.g., Tulving \& Gold, 1963; Reicher, 1969). It is quite probable, then, that the process through which contextual information affects perception is the same for a variety of types of stimuli.

Performance improves as exposure duration increases, though the effect is not as strong as expected. The relatively small influence of exposure duration may be due to the lack of a masking field. If the afterimage is long relative to the actual exposure duration, its effect would tend to mask the small differences in physical exposure.

A three-way analysis of variance (Exposure by Objects by Contextual Conditions) was performed on these data, pooled over subjects and pairings with objects treated as

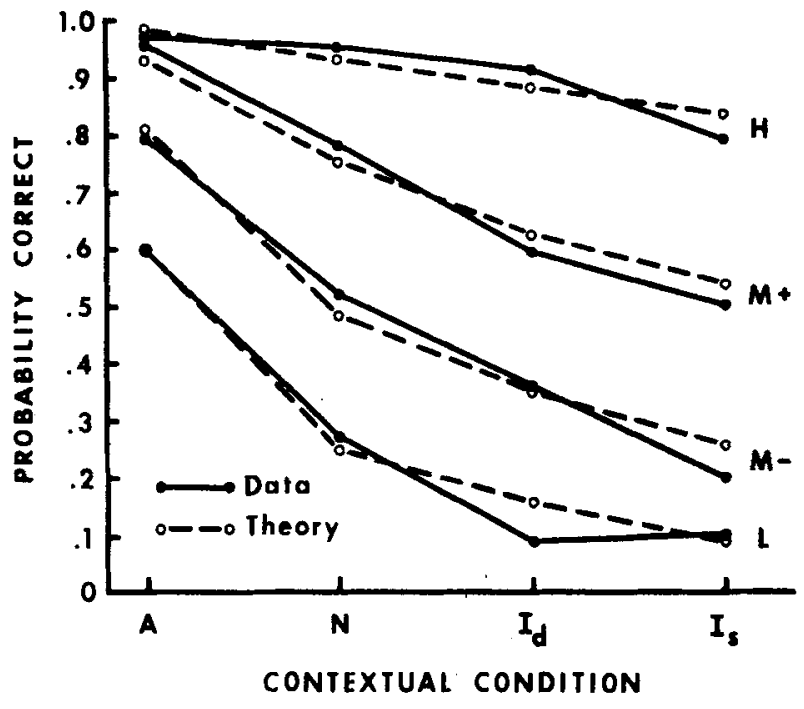

Figure 3. Probability of naming the presented object in each contextual condition ( $A, N, I_{d}$, and $I_{s}$; see text) for four groups of objects based on their overall level of recognizability: high (H), medium high $(\mathrm{M}+)$, medium low $(\mathrm{M}-)$, and low (L). Theoretical curves represent best fit solution of a model for contextual effects.

a random variable. All main effects were significant: exposure, $[F(3 / 69)=9.31, \quad p<.01] ;$ objects, $[F(23 / 1152)=28.97, \quad p<.001] ;$ and contextual conditions, $[\mathrm{F}(3 / 69)=43.30, \mathrm{p}<.001]$. The interaction of objects with contextual conditions was also significant, $[F(69 / 1152)=2.19, \mathrm{p}<.01]$.

It seemed likely that the interaction of objects with contextual conditions was due to a ceiling effect. That is, some of the objects were so recognizable that the different contexts had little effect. To examine this possibility, the 24 objects were rank ordered according to the proportion of correct responses for each object over all exposures and contextual conditions. They were then divided into four groups: high $(\mathrm{H})$, medium high $(\mathrm{M}+)$, medium low (M-), and low (L), based on this measure of overall recognizability. The probability of a correct response was then calculated for each group of objects in each contextual condition. These data are plotted in Figure 3. In addition to the large effect of the different object groups, it is evident that both floor and ceiling effects are responsible for the interaction. The effect due to different objects may be caused by variations in a number of factors: the contrast of the slides, the dimensions of the drawings, the familiarity of the object depicted, or even the "typicality" of the drawing as an instance of the intended object (cf. Rosch, in press).

A second analysis was performed using a four-factor design in which exposure and pairings are factorially combined as between-subjects variables (subjects being 
nested within the Exposure by Pairings design) and contextual conditions as a within-subjects variable. For this analysis, the data were pooled over the objects factor. The results supported those of the previous analysis. Because both subjects and objects are properly considered random variables (see Clark. 1973), min $F^{\prime}$ was calculated for exposure and contextual conditions. Using this test, contextual conditions remained a highly significant factor, $\left[\min F^{\prime}(3 / 103)=29.34, p<.001\right]$. while exposure proved only marginally signiticant, [min $\left.\mathrm{F}^{\prime}(3 / 101)=3.69, \mathrm{p}<.05\right]$.

\section{Within-pair Errors}

The effects of context can be further analyzed by looking at the incorrect responses. In particular, the probability of responding with the name of the other object of the similar pairs is shown in Figure 4. As expected, this kind of error is very unlikely with an appropriate context (Condition A), but is more likely in the other conditions. The probability of a within-pair error when there is no context (Condition N) gives a measure of the visual confusability of the pairs of similar items. Note that the probability of a within-pair error in the $I_{d}$ condition is approximately the same as in the $\mathbf{N}$ condition. Thus, the $I_{d}$ contexts did not bias responses away from this kind of error whereas the A contexts did. The overwhelming effect, though, is that subjects made within-pair errors very frequently in the $I_{s}$ condition where the error is consistent with the context as well as the overall visual characteristics of the presented object. In fact, within-pair errors were made with about the same frequency as correct responses in this condition.

The statistical analyses performed on the within-pair errors were the same as those performed on correct responses. The first analysis (Exposure by Objects by Contextual Conditions, pooled over subjects and

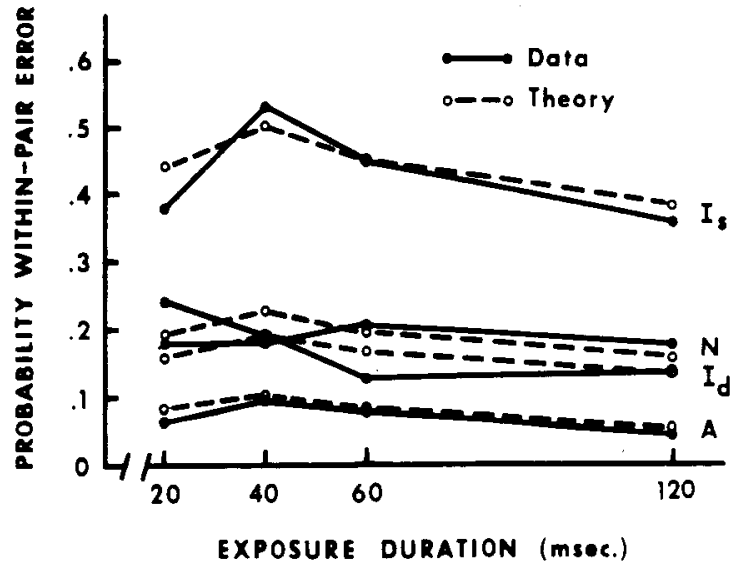

Figure 4. Probability of naming the other object of a visually similar pair as a function of exposure duration for each contextual condition: appropriate context (A), no context $(N)$, inappropriate context, different object $\left(I_{d}\right)$, and inappropriate context, similar object $\left(I_{s}\right)$. Theoretical curves represent best fit solution of a model for contextual effects. pairings) indicated significant main effects for exposure. $[F(3 / 69)=3.13, p<.05]$, objects, $[F(23 / 1152)=21.43, \mathrm{p}<.001]$, and contextual conditions, $[F(3 / 69)=32.33, p<.001]$. Both exposure and objects produced significant interactions with contextual conditions: $[\mathrm{F}(9 / 207)=2.43, \mathrm{p}<.05]$ and $[F(69 / 1152)=2.58, \quad p<.01]$, respectively. The interaction between exposure and contextual conditions is evident in the nonparallelism of the curves in Figure 4. To illuminate the source of the interaction between objects and contextual conditions, the 24 objects were again divided into four groups based on overali recognizability - the same groups used in analyzing the correct responses (see Figure 3). The probability of responding with the name of the other object in the visually similar pair was then calculated for each contextual condition. These data, as plotted in Figure 5, reveal the strong floor effect responsible for the interaction, and illustrate the considerable differences between the particular objects used in the experiment.

The second analysis (including exposure, pairings, subjects, and contextual conditions, pooled over objects) supported the results of the first. Combining the results of the two analyses (with both subjects and objects being treated as random variables) showed that contextual conditions was a highly significant variable, $\left[\min \quad F^{\prime}(3 / 129)=22.33, \quad p<.001\right]$, while neither exposure nor the interaction of exposure with contextual conditions reached statistical significance.

\section{Confidence Ratings}

Confidence ratings were treated in two ways. First, the raw confidence ratings were recorded. Then the confidence ratings were signed $(+$ or -$)$, conditional on the category of the response. In analyzing for correct

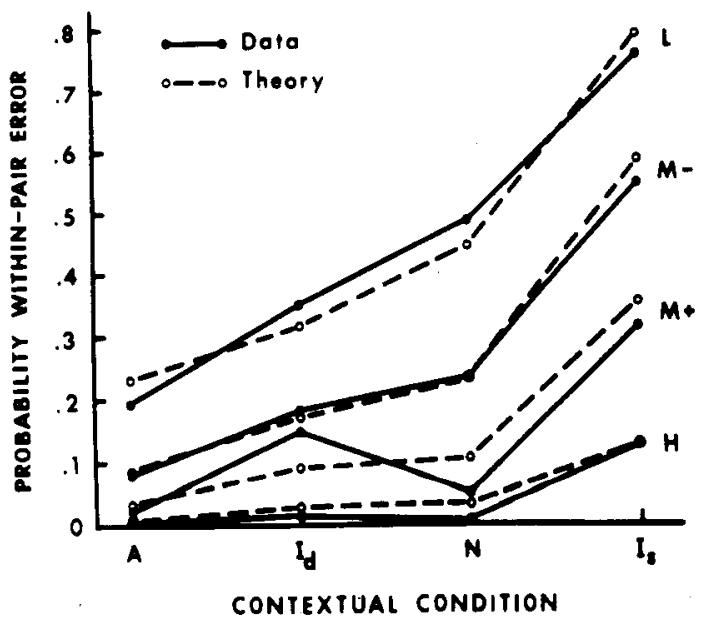

Figure 5. Probability of naming the other object of a visually similar pair in each contextual condition $\left(A, N, I_{d}\right.$, and $I_{s}$; see text) for four groups of objects with different levels of recognizability: high (H), medium high (M+), medium low (M-), and low $(\mathrm{L})$. Theoretical curves represent best fit solution of a model for contextual effects. 
Table 1

Mean Confidence Ratings (and Frequencies) for Response Categories and Contextual Conditions

\begin{tabular}{cccc}
$\begin{array}{c}\text { Contextual } \\
\text { Condition }\end{array}$ & $\begin{array}{c}\text { Correct } \\
\text { Responses }\end{array}$ & $\begin{array}{c}\text { Within-Pair } \\
\text { Errors }\end{array}$ & $\begin{array}{c}\text { Other } \\
\text { Errors }\end{array}$ \\
\hline \multirow{2}{*}{$\mathrm{A}$} & 4.53 & 3.67 & 2.13 \\
& $(319)$ & $(27)$ & $(38)$ \\
$\mathrm{N}$ & 4.15 & 3.28 & 2.10 \\
& $(241)$ & $(72)$ & $(71)$ \\
$\mathrm{I}_{\mathrm{d}}$ & 4.05 & 3.62 & 2.37 \\
& $(188)$ & $(65)$ & $(131)$ \\
& 4.20 & 3.78 & 2.49 \\
$\mathrm{I}$ & $(153)$ & $(166)$ & $(65)$ \\
\hline
\end{tabular}

Vote-Vumbers in parentheses denote response frequencies for the cells.

responses, confidence ratings were multiplied by +1 if the response was correct, and by -1 if the response was a within-pair error or any other error. Similarly, in analyzing for within-pair errors, confidence ratings were multiplied by +1 if the response was a within-pair error. and by -1 if the response was correct or any other error. These signed confidence ratings are, in effect, augmented measures of the probability of correct responses and within-pair errors.

Signed confidence ratings essentially duplicated the pattern of results for response probabilities reported above. Ratings conditionalized on correct responses were highest in the A condition, intermediate in the $\mathrm{N}$ and $\mathrm{I}_{\mathrm{d}}$ conditions, and lowest in the $\mathrm{I}_{\mathrm{s}}$ condition. Confidence ratings conditionalized on within-pair errors were highest in the $I_{s}$ condition, intermediate in the $N$ and $I_{d}$ conditions. and lowest in the A condition. Analyses of variance for signed confidence ratings yielded results nearly identical to those for response probabilities.

Raw confidence ratings were also analyzed by response categories and contextual conditions. Mean raw confidence ratings were calculated for correct responses, within-pair errors, and other errors in each of the four contextual conditions. As indicated in Table 1, confidence ratings are highest for correct responses, lower for within-pair errors, and lowest for other errors. Within response categories, however, the different contextual conditions produce only slight perturbations in confidence ratings. This is in contrast to the large differences between contextual conditions in the frequencies of response types (Table 1, parenthesized numbers). These results indicate that contextual information is important in selecting a response, but not in rating confidence in that response. Once the response has been made, confidence is judged by assessing the "goodness of the fit" between the perceptual data extracted from the stimulus and the perceptual data encoded in the internal representation of the named object. It is important to note, however, that this is a reasonable strategy, given that subjects know the contextual scene might be inappropriate for the presented object.

Statistical analyses support these observations. Approximate analyses of variance were performed on cell means using two designs. In the first analysis. the 64 subjects were divided into four groups (balanced for exposure duration), yielding four observations for each cell in Table 1. In the second analysis, the 24 objects were divided into four groups (balanced for level of recognizability), also yielding four observations per cell. The grouping of subjects and objects was necessitated by low response frequencies in certain cells. The combined results of the two repeated measures analyses show a strong main effect of response category [min $\left.F^{\prime}(2 / 11)=30.08, p<.01\right]$, but no significant effects due to contextual conditions $\left[\mathrm{min} F^{\prime}(3 / 11)=1.47\right.$, $p>$.10] or to the interaction between response categories and contextual conditions $\left[\min \mathrm{F}^{\prime}<1\right]$.

\section{A MODEL FOR CONTEXTUAL EFFECTS}

This experiment shows that both the context and the sensory characteristics of the presented object determine the response made by the subject. A quantitative assessment of this interaction between sensory and contextual information is possible with the "logogen" model developed by Morton (1969, 1970). Morton assumes the existence of internal informational units, called logogens, which are activated by a priori expectations, context, and the similarities of their perceptual encoding with the item presented. In the present experiment, if context $C_{k}$ and sensory signal $S_{j}$ are presented, the activation of the logogen unit $R_{i}$ is determined jointly by the contextual biasing of $C_{k}$ on $R_{i}$, denoted by the variable $c_{k i}$, and the sensory activation of $S_{j}$ on $R_{i}$, denoted by the variable $s_{j i}$. The total activation of a logogen is given by the product of the sources of information; that is, the joint effect of $C_{k}$ and $S_{j}$ on $R_{i}$ would be $s_{j i} c_{k i}$. The probability of selecting a given unit for a response follows Luce's (1959) choice axiom: the probability is the ratio of the activation of a particular unit to the sum of the activations of all units. Thus, for the presentation of context $C_{k}$ and sensory signal $S_{j}$, the probability of response $R_{i}$ would be

$$
P\left(R_{i} \mid C_{k}, S_{j}\right)=\frac{s_{j i} c_{k i}}{\sum_{h=1}^{n} s_{j h} C_{k h}}
$$

where $s, c, n>1$ and $n$ represents the size of the response set.

To test this model against the present data, a number of simplifying assumptions will be made. First, assume that the context $C_{k}$ affects only the logogen $R_{k}$ corresponding to the contextually appropriate object, $\mathrm{S}_{\mathrm{k}}$, defined by the experimenter. Further, assume this 
contextual activation has a constant value, c, for all appropriate object-scene pairs, and a value of 1 (i.e., no effect) for all other pairings. Thus, for example, the kitchen counter scene in Figure 1 exerts an activation of c units on the logogen corresponding to the response "loaf of bread" and activations of 1 on the logogens corresponding to any other response. Second, assume that the stimulus $S_{j}$ affects only two logogens: $R_{j}$ which corresponds to the presented object and $R_{j}$, which corresponds to the other object of the visually similar pair in the experiment. For mathematical simplicity, we further assume that these sensory activations are constant for all presented and within-pair objects, denoted by the parameters $s_{1}$ and $s_{2}$, respectively. Thus, the presentation of the loaf of bread (Figure 1, Drawing A) exerts a sensory activation of $s_{1}$ units on the logogen for "loaf of bread", $s_{2}$ units on the logogen for "mailbox", and 1 unit on all other logogens. Similarly, the presentation of the mailbox (Figure 1, Drawing B) exerts sensory activations of $s_{1}, s_{2}$, and 1 on the logogens for "mailbox," "loaf of bread," and any other response, respectively.

Now, it is easy to derive the equations for the response probabilities in the present experiment. By making the stated simplifications of Equation 1 according to the experimental situations in each of the four contextual conditions, we derive the eight equations required to provide a quantitative assessment of the data for correct responses and within-pair errors. These equations are presented in Table 2.

\section{Exposure Duration and Contextual Conditions}

The first set of data to be predicted is shown in Figures 2 and 4 , representing the effects of exposure duration and contextual conditions on the probabilities of making correct identifications and within-pair errors. There are four basic parameters to the model equations: $s_{1}, s_{2}, c$, and $n$. The sensory parameters, $s_{1}$ and $s_{2}$, are expected to vary with the amount of stimulus information extracted and, therefore, with exposure duration. Thus, we require four separate estimates of $s_{1}$ and $s_{2}$ for the four exposure durations, yielding eight sensory parameters. However, the context parameter, $\mathrm{c}$, and the response set size, $\mathrm{n}$, are expected to remain invariant over different exposure durations. Altogether, then, 10 parameter estimates are required to fit the data. According to the psychological interpretations of the parameters, $s_{1}$ should increase monotonically with exposure duration as more sensory information is extracted. However, $s_{2}$ should begin to decrease (after an initial increase) as specific, discriminating features of the presented object are extracted, thus decreasing the similarity between the extracted information and the encoding of the other object of the visually similar pair.

The 10 model parameters were estimated by a computer application of a minimum chi-square procedure. The best fitting solution, as shown in Figures 2 and 4, exhibits a good match to the data, yielding $\left[\mathrm{X}^{2}(22)=22.90, \mathrm{p}>.40\right.$ and a root mean squared deviation (RMSD) of .036. In addition, the sensory parameters behave in the expected manner: $s_{1}$ increases monotonically with increasing exposure and $s_{2}$ shows an initial increase followed by a decrease at the longest exposure (see Table 3A). The only difficulty with interpreting the parameters is that the lowest chi-square is obtained with the response set size, $n$, estimated between four and five. This value is much too small to represent the number of possible responses. The inconsistency between n's estimated value and its psychological interpretation will be discussed after the second test of the model is presented.

\section{Object Recognizability and Contextual Conditions}

The basic model can also be used to predict the data for the interaction of objects with contextual conditions, as shown in Figures 3 and 5. Recall that these data were compiled by dividing the 24 objects into four groups according to their overall level of recognizability: high $(\mathrm{H})$, medium high $(\mathrm{M}+)$, medium low (M-), and low (L). Response probabilities were then computed for each level of object recognizability within each contextual condition.

The model analysis for these data is similar to that just described. The main change is that different estimates of the sensory parameters are made for each of the four object groups (rather than exposure durations). Thus, there are 10 parameter estimates required to fit the data. Naturally, we expect that the values of $s_{1}$ will increase with increasing recognizability of the object groups. It is also likely (though not necessary) that the values of $s_{2}$ will decrease with increasing recognizability of the object groups.

The 10 parameters were estimated for the 32

Table 2

Equations for Response Probabilities

No Context Condition $(N)$
CR* $\quad P\left(R_{i} \mid C_{0}, S_{i}\right)=s_{1} /\left(s_{1}+s_{2}+n-2\right)$
WPE $\dagger \quad P\left(R_{j} \mid C_{0}, S_{i}\right)=s_{2} /\left(s_{1}+s_{2}+n-2\right)$
Appropriate Context Condition $(A)$
CR $\quad P\left(R_{i} \mid C_{j}, S_{j}\right)=s_{1} c /\left(s_{1} c+s_{2}+n-2\right)$
WPE $\quad P\left(R_{j} \mid C_{i}, S_{i}\right)=s_{2} /\left(s_{1} c+s_{2}+n-2\right)$
Inappropriate Context $/$ Similar Object Condition $\left(I_{s}\right)$
CR $\quad P\left(R_{i} \mid C_{j}, S_{j}\right)=s_{1} /\left(s_{1}+s_{2} c+n-2\right)$
WPE $\quad P\left(R_{j} \mid C_{j}, S_{j}\right)=s_{2} c /\left(s_{1}+s_{2} c+n-2\right)$
Inappropriate Context/Different Object Condition $\left(I_{d}\right)$
CR $\quad P\left(R_{i} \mid C_{k}, S_{j}\right)=s_{1} /\left(s_{1}+s_{2}+c+n-3\right)$
WPE $\quad P\left(R_{j} \mid C_{k}, S_{i}\right)=s_{2} /\left(s_{1}+s_{2}+c+n-3\right)$

Note-Subscripts $i$ and $j$ denote objects (and their associated contextual scenes/ within visually similar object pairs. Subscript $k$ denotes objects (and scenes) outside these pairs. $C_{0}$ is a blank scene.

*Correct responses tWithin-pair errors 
Table 3

Parameter Estimates

A. Exposure Duration and Contextual Conditions

\begin{tabular}{|c|c|c|c|c|c|c|c|c|c|c|c|c|}
\hline $\begin{array}{l}\text { Parameter } \\
\text { Exposure }\end{array}$ & All & $\begin{array}{l}\mathrm{c} \\
\text { All }\end{array}$ & $\begin{array}{r}s_{1} \\
20\end{array}$ & $\begin{array}{r}s_{1} \\
40\end{array}$ & $\begin{array}{r}s_{1} \\
60\end{array}$ & $\begin{array}{r}s_{1} \\
120\end{array}$ & $\begin{array}{c}\mathrm{s}_{2} \\
20^{2}\end{array}$ & $\begin{array}{r}s_{2} \\
40\end{array}$ & $\begin{array}{c}s_{2} \\
60^{2}\end{array}$ & $\begin{array}{r}s_{2} \\
120^{2}\end{array}$ & $x^{2}$ & RMSD \\
\hline Estimate & 4.53 & 3.37 & 5.22 & 5.73 & 9.52 & 10.33 & 1.83 & 2.41 & 2.87 & 2.32 & $22.90^{*}$ & .036 \\
\hline \multicolumn{13}{|c|}{ B. Object Groups and Contextual Conditions } \\
\hline $\begin{array}{l}\text { Parameter } \\
\text { Obj. Group }\end{array}$ & $\begin{array}{l}\mathrm{n} \\
\text { All }\end{array}$ & $\begin{array}{l}\mathrm{c} \\
\text { All }\end{array}$ & $\begin{array}{l}\mathrm{s}_{1} \\
\mathrm{H}\end{array}$ & $\frac{s_{1}}{M+}$ & $\begin{array}{l}s_{1} \\
M-\end{array}$ & $\begin{array}{l}s_{t} \\
L\end{array}$ & $\begin{array}{l}\mathrm{s}_{2} \\
\mathrm{H}\end{array}$ & $\begin{array}{l}s_{2} \\
M+\end{array}$ & $\begin{array}{l}\mathrm{s}_{2} \\
\mathrm{M}-\end{array}$ & $\begin{array}{l}\mathbf{s}_{2} \\
\mathrm{~L}\end{array}$ & $x^{2}$ & RMSD $\dagger$ \\
\hline Estimate & 4.98 & 4.69 & 63.28 & 14.39 & 4.84 & 2.23 & 2.00 & 2.00 & 2.31 & 4.00 & $30.60 * *$ & .034 \\
\hline
\end{tabular}

experimental points using a minimum chi-square procedure. Inspection of Figures 3 and 5 shows that the best fitting solution of the model again matches the data quite closely, RMSD $=.034$. The statistical fit for these data, $\left[X^{2}(22)=30.60, p>.10\right]$, is not as good as for the previous data, but the difference is due to the larger number of points approaching the extreme probabilities of zero and one.

As expected, the $s_{1}$ parameter increases substantially with increasing recognizability of the object groups (see Table 3B). In contrast, the $s_{2}$ parameter remains relatively constant, increasing only for the least recognizable group of objects $(L)$. The fact that the estimate of $s_{2}$ is greater than that of $s_{1}$ is not troublesome because the "sensory parameters" $s_{1}$ and $s_{2}$ in this simplified application of Morton's model actually incorporate any a priori biases which exist (due to frequency, for example). In comparing the two analyses summarized in Table 3, note that the estimates of the response-set size, $\mathrm{n}$, and contextual activation, $\mathrm{c}$, agree well.

\section{Size of the Response Set}

The estimated value of $n$ is approximately five in both analyses. This is a significant problem for a model in which $n$ represents the total number of responses. One possible explanation is that the model may be quite insensitive to the size of $n$, even though the best fitting estimate is small. Additional analyses, however, showed that acceptable fits could not be obtained with n fixed at values large enough to be interpreted as the number of possible responses. Antother possibility is that the assumptions made in simplifying the general model are responsible for the low estimate of the response set size.

A simpler solution to the problem, however, is to reinterpret $\mathrm{n}$ as the size of the "candidate set" as suggested by Rumelhart (in press; Rumelhart \& Siple, 1974). With this interpretation, $n$ represents the number of responses under consideration after eliminating responses which are contradicted or unlikely given the information gathered to that point. The good fit of the model to the present data with a low value for $n$ presumably indicates that at the exposure durations used (without a masking field), enough sensory information has been gathered to eliminate all but a few of the possible responses.

Given the high ratio of model parameters to data points, the success of the model must be interpreted with some caution. I do not claim that the logogen model is the only one which can account for the present data or even that it is the best model for these data. However, it has been applied to a variety of contextual effects with creditable success (Morton, 1969, 1970). The present results simply add to existing evidence that this type of formulation is viable as an abstract characterization of how contextual and sensory data are integrated during perceptual identification.

A more complete characterization of perceptual identification requires the elaboration of the abstract model in terms of information structure and processing. "Logogens" may be further specified in terms of their representational format (features, propositions, or whatever) and informational content. The relationships between particular logogens encode specific world knowledge-e.g., that a loaf of bread is likely to be located on a counter in a kitchen and that a mailbox is likely to be located in the front yard of a house. These relational interconnections, then, are the medium through which contextual information exerts its influence on perceptual interpretation. An information processing model of perception along these lines is presented in Palmer (in press).

\section{REFERENCES}

Biederman, I. Perceiving real world scenes. Science, 1972, 177, $77-80$.

Biederman, I. , Glass, A. L. \& Stacy, E. W. Searching for objects in real world scenes. Journal of Experimental Psychology, $1973,97,22-27$

Bruner, J. S. On preceptual readiness. Psychological Review, $1957,64,123-152$.

Clark, H. H. The language-as-fixed-effect fallacy: Critique of language statistics in psy chological research. Journal of Verbal Learning and Verbal Behavior, 1973, 12, 335-359.

Luce, R. D. Individual choice behavior. New York: Wiley, 1959. Morton, $J$. Interaction of information in word recognition. Psychological Review, 1969, 76, 165-178.

Morton, $j$. A functional model for memory, In D. A. Norman (Ed.), Models of human memory. New York: Acadernic Press, 1970 .

Neisser, U. Cognittue psychology. New York, Appleton-Century Crofts, 1967.

Norman, D. A. Toward a theory of memory and attention. Psychological Review, 1968, 75, 522-536. 
Palmer, S. E. Visual perception and world knowledge: Notes on a model of sensory-cognitive interaction. In D. A. Norman, D. E. Rumelhart, \& the LNR Research Group, Explorations in cognition. San Francisco: W. H. Freeman, in press.

Reicher, G. M. Perceptual recognition as a function of meaningfulness of stimulus materials. Journal of Experimental Psychology, 1969, 81, 275-280.

Rosch, E. H. Universals and culture specifics in human categorization. In R. Brislin, S. Bochner, \& W. Lonner (Eds.), Crose-cultural perspectives on learning. Sage Press, in press.

Rumelhart, D. E. A multicomponent theory of confusions among briefly exposed alphabetic characters. Perception \& Psychophysics, in press.

Rumelhart, D. E. and Siple, P. A. The process of recognizing tachistoscopically presented words. Psychological Review, $1974,81,99-118$.
Tulving, E., \& Gold, C. Stimulus information and contextual information as determinants of tachistoscopic recognition of words. Joumal of Experimental Psychology, 1963, 66, 319-327.

Tulving, E., Mandler, G. \& Baumel, R. Interaction of two sources of information in tachistoscopic word recognition. Canadian Joumal of Psychology, 1964, 18, 62-71.

Wheeler, D. D. Processes in word recognition. Cognitive Psychology, 1970, 1, 59-85.

(Received December 21, 1973 .

Revision received November $18,1974$. ) 